\title{
ANÁLISIS DEL TEXTO DE LA CONSTITUCIÓN A LA LUZ DEL POSDCORB*
}

\section{ANALYSIS OF THE TEXT OF THE CONSTITUTION IN LIGHT OF THE POSDCORB}

\section{ANÁLISE DO TEXTO DA CONSTITUÇAO À LUZ DO POSDCORB}

Mariel Lorenzo Pena**

RESUMEN. En tanto el derecho no es una materia aislada, el estudio del texto constitucional a partir de conceptos vertidos por otras ciencias y materias resulta interesante. En este contexto, se propone un análisis de nuestro máximo texto normativo desde el aporte de la ciencia de la administración. En esta óptica, se definen y estudian los conceptos de Planning, Organizing, Staffing, Directing, Co-Ordinating, Reporting and Budgeting (en español planificación, organización, dotación de personal, dirección, coordinación, informes y presupuestos) los que se reúnen en el acrónimo POSDCORB.

Luego, se propone realizar una primera lectura de nuestra Carta Magna a la luz de estos conceptos. Se expone brevemente qué artículos de nuestro texto constitucional podrían ingresar en cada uno de ellos. Esta lectura se realiza en base a palabras exactas encontradas en el texto superior (resultados literales de búsquedas) así como ingresando al contenido de algunos artículos de la Carta, categorizando los mismos en base a este mismo criterio.

PALABRAS CLAVE. POSDCORB. Constitución. Ciencia de la Administración.

ABSTRACT. Inasmuch as the law is not an isolated subject, the study of the constitutional text from concepts discharged by other sciences and matters is interesting. In this context, an analysis of our maximum normative text from the contribution of the science of administration is proposed. In this perspective, the concepts of Planning, Organizing, Staffing, Directing, Co-Ordinating, Reporting and Budgeting -collected in the acronym POSDCORB- are defined and studied. Then, it is proposed to make a first reading of our Magna Carta in light of these concepts. The articles of our constitutional

\footnotetext{
* Planning, Organizing, Staffing, Directing, Co-Ordinating, Reporting and Budgeting (POSDCORB).

El presente artículo fue realizado como parte de la aprobación del curso «Organización Administrativa» a cargo del Prof. Felipe Rotondo en el contexto del LL.M -Máster en Derecho- de la Universidad de Montevideo. Se cuenta con la autorización de la mencionada Universidad para realizar esta publicación. La autora agradece especialmente al Prof. Pablo Genta, Coordinador Académico de Máster y Postgrados por dicha autorización.

** Doctora en Derecho y Ciencias Sociales por la UdelaR. Profesora Adscripta en Derecho Constitucional por la UdelaR. Cursando Maestría LL.M. -Máster en Derecho- por la Universidad de Montevideo. Profesora Asistente (Grado II) de Derecho Constitucional (UdelaR). Correo electrónico: mariel.lorenzo.pena@gmail.com
} 
text that could enter into each of those concepts are briefly exposed. This reading is made based on exact words found in our higher text (literal search results) as well as entering the content of some articles of the Charter, categorizing them based on this same criterion.

KEY WORDS. POSDCORB. Constitution. Science of Administration.

RESUMO. Enquanto o direito não é um assunto isolado, o estudo do texto constitucional a partir de conceitos expressos por outras ciências e assuntos é interessante. Nesse contexto, uma análise de nosso texto normativo máximo é proposta a partir da contribuição da ciência da administração. Nessa perspectiva, são definidos e estudados os conceitos de planejamento, organização, pessoal, direção, coordenação, elaboração de relatórios e orçamento; ou seu acrônimo POSDCORB.

Em seguida, propõe-se fazer uma primeira leitura de nossa Magna Carta à luz desses conceitos. Expõem-se brevemente quais artigos de nosso texto constitucional poderiam entrar em cada um deles. Essa leitura é feita com base nas palavras exatas encontradas no texto superior (resultados literais da pesquisa), bem como na inserção do conteúdo de alguns artigos da Carta, categorizando-os com base nos mesmos critérios.

PALAVRAS-CHAVES. POSDCORB. Constituição. Ciência da Administração.

\section{INTRODUCCIÓN}

"No deberá olvidar ni por un solo instante que la materia prima con que trabaja la administración la constituyen seres humanos" Urwick

Estudiar derecho implica adentrarse también en otras disciplinas, sociología, ciencia administrativa, economía y hasta psicología. Ello porque, concebido aisladamente, el derecho pierde sentido.

Esta necesaria interdisciplinariedad conlleva el interés por conceptos que provienen de otras áreas, pero que se hacen aplicables al derecho. Particular interés despierta la ciencia de la administración, la que según Delpiazzo (SF:832), en una de sus posibles acepciones, se ha entendido como «el estudio de los hechos administrativos y, en particular, la esencia de la acción dinámica de la Administración (en su desarrollo histórico y en sus soluciones comparadas), la explicación de cómo esa acción se desarrolla y realiza, y la descripción 
de "lo que se hace" en todos los sectores de la actividad administrativa».

El tema de este trabajo es el «Análisis del texto constitucional a la luz del POSDCORB».

El objetivo en estas breves páginas es referenciar los conceptos en sí vertidos en el acrónimo POSDCORB -encuadrando teóricamente el tema-, para posteriormente identificar -en una primera lectura- los artículos de nuestra Constitución que reflejan alguno o algunos de los aspectos introducidos por el concepto mencionado, tanto desde el punto de vista literal, como desde lo conceptual.

El análisis para la categorización debe ser cuidadoso, dado que el constituyente no emplea el lenguaje de la ciencia administrativa. Es necesario advertir además que diferentes partes de un artículo pueden ser incluidas en categorías diferentes.

Se pretende simplemente dar una mirada diferente a nuestro texto constitucional, siempre tan profundo como multifacético. Mirada que, por ser propia, será necesariamente subjetiva y por tanto, incompleta.

\section{ENCUADRE TEÓRICO}

Planning, Organizing, Staffing, Directing, Co-Ordinating, Reporting and Budgeting (POSDCORB), o lo que es lo mismo en español, planificación, organización, dotación de personal, dirección, coordinación, informes y presupuestos.

El POSDCORB es un concepto perteneciente al ámbito de la administración pública, que marca los pasos necesarios en el proceso administrativo.

Henry Fayol -célebre ingeniero de minas y principal exponente de la teoría clásica- introduce en 1916 la distinción entre Administración y Organización. A partir de sus obras -y la de varios de sus seguidores-, «la atención se dirige hacia la organización del trabajo y de la producción en procura de eficiencia y rendimiento» (Delpiazzo, SF:822).

La administración es un todo del cual la organización es una de las partes, mientras que la segunda refiere solamente al establecimiento de la estructura y de la forma, por lo tanto es estática y limitada (Riascos, SF).

Nos dice Fayol (1961:136) que «ADMINISTRAR, es prever, organizar, dirigir, coordinar y controlar; Prever, es decir escrutar el futuro y articular el programa de acción; Orga- 
nizar, es decir constituir el doble organismo, material y social, de la empresa; Dirigir, es decir hacer funcionar el personal; Coordinar, es decir relacionar, unir, armonizar todos los actos y todos los esfuerzos; Controlar, es decir procurar que todo se desarrolle de acuerdo con las reglas establecidas y las órdenes dadas».

Conforme Guerrero (1997:179) el concepto de administración en su sentido más amplio, tiene un significado más general que lo relativo a la vida pública, es decir, la administración del Estado. Por otra parte, no es que exista una Administración, sino que existe la administración de la iglesia, del culto, la administración pública. La diferencia estriba en que «dentro de este abanico de administraciones, la administración privada es considerada como un deber de la familia, de la asociación o de una religión, pero nunca como un deber hacia el Estado. De la administración pública se encargan funcionarios, de la privada los gerentes» (Guerrero, 1997:179).

Y esto porque, no debe olvidarse, «el Estado es, fundamentalmente, una organización que ejerce el poder en aras del interés general, el cual en un Estado de Derecho se centra en el pleno desarrollo del ser humano» (Rotondo, 2015:127).

De acuerdo a lo expuesto por Guerrero (1997:177), el «estudio de la administración pública como la actividad del Estado, permite rebasar las visiones introspectivas y gerenciales del Poder Ejecutivo, frecuentemente inclinadas a equiparar el proceso administrativo interno del gobierno, con el proceso administrativo de la empresa privada». «La clave de la distinción de esfera de naturaleza entre la administración pública y la administración privada, se encuentra en la relación entre los intereses generales y los intereses particulares. [...] Si consideramos que la nación es un organismo político, y la sociedad el conjunto de las clases y los individuos, entonces la diferencia de los intereses de ambos es muy importante porque la nación y la sociedad tienen relaciones estrechas: "el Estado goza del bienestar de la sociedad y padece sus males, y la sociedad necesita con frecuencia del auxilio del Estado. Pero la oposición no es radical, por lo que el estadista debe poner sus ojos igualmente en los intereses generales y los intereses individuales, atendiendo a esas importantes y diversas cuestiones que mencionamos, y que se encuentran en ambos» (Guerrero, 1997:181).

Dentro de los principios que rigen la administración enumera Fayol (1961:156) los siguientes: la división del trabajo, la autoridad, la disciplina, la unidad de mando, la unidad de dirección, la subordinación de los intereses particulares al interés general, la remuneración, la centralización, la jerarquía, el orden, la equidad, la estabilidad del personal, la iniciativa y la unión del personal.

Por otra parte, «la Organización, considerada estrictamente en su sentido limitado, podría 
definirse muy sencillamente como: “determinar cuáles son las actividades necesarias para cualquier propósito (o "plan"), disponiéndolas en grupos que puedan asignarse a individuos» (Urwick, 1967:47). Quiere decir esto que organizar implica dividir y especializar, de forma de aumentar la eficiencia en el trabajo.

La especialización puede hacerse de cuatro formas: de acuerdo al fin al que los trabajadores sirven, de acuerdo al proceso que los trabajadores utilizan, de acuerdo a la clientela o material que los trabajadores tratan y de acuerdo al lugar donde se realiza el trabajo. Vemos aquí como la división del trabajo se relaciona y condiciona en parte a la organización -y viceversa-.

Las funciones del administrador y la clasificación de Fayol, serán profundizadas en 1937 por Luther Gulick y Lyndall Urwick al publicar "Papers on the Science of Administration” (Escritos sobre la Ciencia de la Administración) (Rodríguez, 1999:19).

Tomando ideas de la teoría de la organización militar -VA Graicunas y Sir Ian Hamilton-, estos autores incorporarán la administración por objetivos, dividiendo las funciones del gerenciamiento en las siguientes:

i) La planificación, que implica el trazado de metas y la fijación de procesos y métodos. El proyecto es lo primero, este concepto nos dice Urwik (1967:49) viene de la práctica ingenieril.

ii) La organización, que refiere al establecimiento de la estructura en pos del objetivo buscado. Se aplicará aquí la teoría de la unidad de mando, por la que cada trabajador debe tener sólo un superior directo con el fin de evitar la confusión y la ineficiencia. «Cuando los obreros son reunidos en cuadrilla, cada uno de ellos se vuelve mucho menos eficiente que cuando su ambición personal es estimulada» (Taylor, 1975:53).

iii) La dotación de personal o asesoría, que incluye la función de preparar y entrenar al personal, así como su reclutamiento, conservación y motivación. Se busca la especialización, bajo el lema de «una sola función para cada uno». Se resalta la importancia de las remuneraciones, la disciplina, las sanciones, los ascensos. Dicen los autores que es importante además valorar la iniciativa y regirse siempre con moral y equidad.

iv) La dirección, que estriba en la función de liderar. Debe existir aquí un balance entre el principio de autoridad y el principio de delegación. «El mando debe representar un objetivo común» (Urwick, 1967:105). 
v) La coordinación, que pretende establecer relaciones en las diferentes partes del trabajo. El fin de la organización es asegurar que la división del trabajo funcione correctamente, que haya unidad de esfuerzos (Urwick, 1967:57). Cualquier administrador tendrá una cantidad limitada de tiempo y energía, lo que determinará un máximo de subordinados que puede manejar. Este número depende de factores como la estabilidad de la organización, el espacio en que la actividad se realiza, la especialización de los subordinados y si sus dirigidos provienen del mismo campo o especialidad.

vi) Los informes, en la relación con la potestad y necesidad de mantenerse informado, que están referenciados tanto al personal como a la dirección.

vii) El presupuesto, que refiere tanto a la contabilidad y control, como a la planificación fiscal. Cabe incluir aquí inventarios, balances, cuentas, estadísticas, estimación de costos y gastos efectivos.

Gulick y Urwick «fueron unos teóricos organizativos inusuales, y más para su tiempo, en el sentido que sus estudios empíricos se centraron en organizaciones de naturaleza pública. De esta manera abordaron temas tan importantes para los estudiosos de las administraciones públicas como son las relaciones, siempre críticas, entre la dimensión política y la dimensión funcionarial. Es decir, las tensiones y fricciones entre el político y el profesional» (Ramió, SF:9).

Si bien sus conceptos ya habían sido esbozados por Fayol, el aporte de estos autores estriba en que de manera transparente, señalaron que «si se aceptan estos siete elementos como los principales deberes de un directivo, se deriva de ello que deben ser organizados de manera independiente como subdivisiones del ejecutivo» (Guerrero, 1997:176) lo que dependerá del tamaño y complejidad de la empresa.

\section{ANÁLISIS DEL TEXTO CONSTITUCIONAL}

Esbozadas las categorías que propone el PODSCORB se expondrá brevemente qué artículos de nuestro texto Constitucional podrían ingresar en cada una de éstas.

En un primer estudio -y utilizando las herramientas informáticas con que se cuenta hoy en día-se realizaron búsquedas de palabras exactas en el texto constitucional (resultados literales). 
Posteriormente, se realizó un análisis del contenido de alguno de los artículos de la Carta, categorizando los mismos en base a este criterio.

\section{III.1 PLANIFICACIÓN}

Realizando la búsqueda por la palabra «planif», se observa que los artículos que contienen este concepto son el 47 y el 230.

El artículo 47 refiere al derecho al medio ambiente y establece que «los usuarios y la sociedad civil, participarán en todas las instancias de planificación, gestión y control de recursos hídricos; estableciéndose las cuencas hidrográficas como unidades básicas»». Como se observa, se utiliza aquí el concepto planificación en el sentido esbozado de visualización de un fin, en este caso, la correcta utilización de los recursos hídricos.

Por su parte, el artículo 230 refiere a la Oficina de Planeamiento y Presupuesto (OPP) y establece que ella «asistirá al Poder Ejecutivo en la formulación de los planes y programas de desarrollo, así como en la planificación de las políticas de descentralización que serán ejecutadas» por diferentes actores.

De la simple lectura resulta que el concepto también es utilizado en el sentido esbozado. Más aún en este caso, la planificación está asociada a la existencia misma de una Oficina cuya competencia refiere en particular a esta categoría. Tenemos aquí un claro ejemplo de cómo la administración condiciona la organización, al punto que - a criterio del constituyente-, es necesario contar con una estructura fija y determinada (como es la OPP) para planificar en materia económica (esto se une además con otro concepto del POSDCORB que es el presupuesto, lo que se verá más adelante).

Desde otro punto de vista, es importante tener en cuenta que el artículo en concreto establece que la Oficina de Planeamiento y Presupuesto asiste al Poder Ejecutivo en la planificación, por lo que en puridad podría cuestionarse quién es en verdad el que planifica. No debe olvidarse que conforme el mismo 230 la OPP depende directamente de la Presidencia de la República, cuya posición institucional es discutida. El caso se complejiza aun más si se considera la situación de los entes descentralizados. En el marco del artículo 197 podría el Poder Ejecutivo considerar inconveniente un acto de los directorios siempre que no se ajuste a la planificación realizada.

\section{III.2 DOTACIÓN DE PERSONAL}

La búsqueda realizada refirió a los vocablos «personal» y «empleo». 
De la misma, surge el artículo 56 que refiere a las empresas en general (y no en particular al Estado): «Toda empresa cuyas características determinen la permanencia del personal en el respectivo establecimiento, estará obligada a proporcionarle alimentación y alojamiento adecuados, en las condiciones que la ley establecerá».

Particular interés para esta categoría representan los artículos 59 a 66.

El artículo 59 indica que «la ley establecerá el Estatuto del Funcionario sobre la base fundamental de que el funcionario existe para la función y no la función para el funcionario». Este artículo puede leerse como indicativo de una relación entre la estructura (la función) y el funcionario, pero puede además leerse en relación a las tareas o especialización (nuevamente la función) que debe adquirir cada uno de los funcionarios, para el desarrollo de su actividad.

Por otra parte, el artículo 60 establece la creación del Servicio Civil, por lo que nuevamente a criterio del constituyente, es necesaria una estructura determinada para sustentar un elemento del POSDCORB.

En agregado, el mismo artículo ya determina ciertas particularidades referentes al personal, dado que garantiza la carrera administrativa y declara a ciertos funcionarios inamovibles, estableciendo determinadas garantías para disponer su destitución. Lo mismo ocurre con la previsión establecida en el artículo 66 en cuanto a que «ninguna investigación parlamentaria o administrativa sobre irregularidades, omisiones o delitos, se considerará concluida mientras el funcionario inculpado no pueda presentar sus descargos y articular su defensa».

El contenido que -conforme el artículo 61 de la Carta- debe tener el Estatuto del Funcionario (condiciones de ingreso, derecho a la permanencia en el cargo, al ascenso, al descanso semanal y al régimen de licencia anual y por enfermedad; condiciones de la suspensión o del traslado; obligaciones funcionales y recursos administrativos contra las resoluciones que los afecten) es claramente el indicado por la S (Staff) del POSCORB.

En relación con lo que disponen los artículos 62 y 63 puede pensarse que es el tipo de actividad (industrial y comercial) que realizan ciertas personas jurídicas, lo que condiciona su estructura y por esto, el constituyente parece creer necesario, que en las mismas, existan estatutos especiales para sus funcionarios. Nuevamente por tanto, se vislumbra la organización (producto de la actividad) influyendo en este concepto. 
Sin perjuicio de esto, y puede entenderse que en aplicación de otro concepto esbozado (la coordinación) establece el artículo 64 que «la ley, por dos tercios de votos del total de componentes de cada Cámara, podrá establecer normas especiales que por su generalidad o naturaleza sean aplicables a los funcionarios de todos los Gobiernos Departamentales y de todos los Entes Autónomos, o de algunos de ellos, según los casos».

En el artículo 65 por otra parte, se vislumbran varios elementos de análisis. El mismo establece:

«La ley podrá autorizar que en los Entes Autónomos se constituyan comisiones representativas de los personales respectivos, con fines de colaboración con los Directores para el cumplimiento de las reglas del Estatuto, el estudio del ordenamiento presupuestal, la organización de los servicios, reglamentación del trabajo y aplicación de las medidas disciplinarias.

En los servicios públicos administrados directamente o por concesionarios, la ley podrá disponer la formación de órganos competentes para entender en las desinteligencias entre las autoridades de los servicios y sus empleados y obreros; así como los medios y procedimientos que pueda emplear la autoridad pública para mantener la continuidad de los servicios».

Por un lado, se observa que pueden crearse comisiones de funcionarios, lo que claramente afecta la estructura. Por otro lado, estas comisiones tienen como fin la colaboración (otro concepto del POSDCORB). Por último, en el caso de servicios públicos, también se ve afectada la estructura dado que se pueden crear órganos, siendo el objetivo de estos la coordinación, pero también la planificación (medios y procedimientos para la continuidad de los servicios).

«Todo ciudadano puede ser llamado a los empleos públicos». En su comienzo, el artículo 76 establece ya cierta condicionante para ciertos miembros del staff del Estado.

El artículo 77 utiliza el término empleo, al establecer como pena accesoria a ciertas conductas la inhabilitación para el ejercicio de ciertos empleos públicos.

En forma más escueta - pero también en relación con el staff-, el artículo 107 establece cada Cámara nombrará a sus secretarios y al personal de su dependencia. Lo mismo ocurre en el artículo 204, el que consagra que los Consejos Directivos establecerán los estatutos en sus respectivos entes de enseñanza.

El análisis nos lleva luego al artículo 168, que establece las competencias del Poder Ejecutivo. Puede considerarse que varios de sus numerales refieren a la dotación del perso- 
nal, así:

$\left.\ll 3^{\circ}\right)$ Dar retiros y arreglar las pensiones de los empleados civiles y militares conforme a las leyes».

$\left.« 9^{\circ}\right)$ Proveer los empleos civiles y militares, conforme a la Constitución y a las leyes». «10) Destituir los empleados por ineptitud, omisión o delito, en todos los casos con acuerdo de la Cámara de Senadores o, en su receso, con el de la Comisión Permanente, y en el último, pasando el expediente a la Justicia. Los funcionarios diplomáticos y consulares podrán, además, ser destituidos, previa venia de la Cámara de Senadores, por la comisión de actos que afecten su buen nombre o el prestigio del país y de la representación que invisten. Si la Cámara de Senadores o la Comisión Permanente no dictaran resolución definitiva dentro de los noventa días, el Poder Ejecutivo prescindirá de la venia solicitada, a los efectos de la destitución».

«11) Conceder los ascensos militares conforme a las leyes, necesitando, para los de Coronel y demás Oficiales superiores la venia de la Cámara de Senadores o, en su receso, la de la Comisión Permanente».

«12) Nombrar el personal consular y diplomático, con obligación de solicitar el acuerdo de la Cámara de Senadores, o de la Comisión Permanente hallándose aquélla en receso, para los Jefes de Misión [...]».

«13) Designar al Fiscal de Corte y a los demás Fiscales Letrados de la República, con venia de la Cámara de Senadores o de la Comisión Permanente en su caso, otorgada siempre por tres quintos de votos del total de componentes. La venia no será necesaria para designar al Procurador del Estado en lo Contencioso-Administrativo, ni los Fiscales de Gobierno y de Hacienda».

«14) Destituir por sí los empleados militares y policiales y los demás que la ley declare amovibles».

«26) El Presidente de la República designará libremente un Secretario y un Prosecretario, quienes actuarán como tales en el Consejo de Ministros. Ambos cesarán con el Presidente y podrán ser removidos o reemplazados por éste, en cualquier momento».

Por otra parte, compete a la Asamblea General (artículo 85 numeral 13) «crear o suprimir empleos públicos, determinando sus dotaciones o retiros, y aprobar, reprobar o disminuir los presupuestos que presente el Poder Ejecutivo; acordar pensiones y recompensas pecuniarias o de otra clase y decretar honores públicos a los grandes servicios». En concordancia con esta previsión, el artículo 86 refleja un impacto en la estructura organizativa, dado que estas creaciones y supresiones de empleo necesariamente impactan en las estructuras de cargos y funciones de las organizaciones estatales.

Con relación a los magistrados y personal del Poder Judicial, el artículo 252 establece 
ciertas prohibiciones como «dirigir, defender o tramitar asuntos judiciales, o intervenir, fuera de su obligación funcional, de cualquier modo en ellos, aunque sean de jurisdicción voluntaria» siendo la pena establecida la destitución inmediata. Vemos aquí que se encuentra regulado en parte también el estatuto de estos funcionarios.

Relacionado al personal, pero a aquel que está encargado de su gestión y dirección, encontramos diferentes previsiones, en cuanto a la cantidad de personas que se requieren en cada caso.

Así, conforme el artículo 185 de la Carta, los directores de entes descentralizados podrán componerse de tres o cinco miembros, mientras que el número de miembros de las autoridades locales puede variar, siendo unipersonales o pluripersonales, conforme lo establecido por el artículo 287.

El estatuto de los directores se encuentra en parte ya previsto en el texto constitucional en sí. Tal es el caso de los artículos 187 y 192 en cuanto a su designación o elección y cese. En cuanto a los Entes de Enseñanza, debemos atenernos a lo dispuesto por el artículo 203. El artículo 289 regula las incompatibilidades para el cargo del Intendente.

En cuando a los legisladores, también se encuentra previsto su estatuto en el texto en estudio. Así la Cámara de Representantes se compone de noventa y nueve miembros (artículo 88) mientras que la de Senadores se compone de treinta miembros y el Vicepresidente de la República (artículo 94).

Los artículos 89 a 92 establecen las inelegibilidades e incompatibilidades para ser Representantes, mientras que lo suyo hacen los artículos 95 a 101 respecto de los Senadores. En cuanto a las prohibiciones, las mismas están previstas para ambas figuras en los artículos 122 a 126.

En relación a los Ministros, los artículos 183 y 184 regulan su designación, licencias y otros aspectos, mientras que el artículo 176 establece las calidades necesarias y el 178 las inmunidades de las que gozan.

La disposición transitoria y especial letra $\mathrm{O}$, también referencia el personal, en este caso de la anterior Comisión de Inversiones y Desarrollo Económico, que pasaba a integrar la recién creada en ese entonces OPP.

Por último, la disposición transitoria y especial letra J utiliza el vocablo personal, pero en relación a la notificación en el marco del Tribunal de lo Contencioso Administrativo, por lo que el sentido utilizado es referido a la persona y no a los trabajadores, empleados, funcionarios. 


\section{III.3 DIRECCIÓN}

El primer artículo que en el texto constitucional utiliza el término «dirección» es el 24, «confiados a su gestión o dirección». Puede verse en el comienzo entonces, que el balance entre autoridad y delegación buscado por el constituyente, parece resolverse con la responsabilidad especialmente consagrada.

El inciso 4 del artículo 188, refiriéndose a la participación estatal en actividades industriales, agropecuarias o comerciales, de empresas formadas por aportes obreros, cooperativos o capitales privados, establece que «la ley, por mayoría absoluta del total de componentes de cada Cámara, autorizará en cada caso esa participación, asegurando la intervención del Estado en la dirección de la empresa».

El artículo 221 referido al presupuesto de los Entes industriales y comerciales establece que «la ley fijará, previo informe de los referidos Entes y del Tribunal de Cuentas y la opinión del Poder Ejecutivo emitida con el asesoramiento de la Oficina de Planeamiento y Presupuesto, los porcentajes que cada Ente podrá destinar a sueldos y gastos de dirección y de administración».

La disposición transitoria y especial letra E utiliza el vocablo dirección pero en referencia a Direcciones de diferentes Ministerios que son creadas por la disposición mencionada. Vemos aquí un caso en el que la dirección incluye en la organización al punto de generar órganos destinados a esta función.

En similar sentido podría asociarse el concepto en el artículo 279 que establece que «el Intendente determinará la competencia de las direcciones generales de departamento y podrá modificar su denominación»».

\section{III.4 COORDINACIÓN}

En este caso la búsqueda realizada fue «coordina». Este concepto es quizás el más vinculado al control y se encuentra en plena relación con el de dirección.

El artículo 202 menciona que la coordinación de la enseñanza se hará por ley.

El artículo 195 establece que uno de los cometidos del Banco de Previsión Social (BPS) es coordinar los servicios estatales de previsión social y organizar la seguridad social.

El final del artículo 262 establece que «habrá un Congreso de Intendentes, integrado por quienes fueren titulares de ese cargo o lo estuvieren ejerciendo, con el fin de coordinar las 
políticas de los Gobiernos Departamentales. El Congreso, que también podrá celebrar los convenios a que refiere el inciso precedente, se comunicará directamente con los Poderes del Gobierno».

La influencia entonces de la coordinación en la organización surge de la simple lectura de estos artículos y vaya si condicionarán la estructura y organización estatal, al punto que crea directamente el texto dos organismos destinados a ello.

La introducción a nivel Constitucional del BPS se estableció en el texto de 1967, mientras que la creación del Congreso de Intendentes se consagró en la reforma de 1996, por lo que podría hacerse notar que la importancia de la coordinación es en cierta forma la novedad del POSDCORB en materia constitucional. Mediante un razonamiento opuesto, podría sostenerse que la coordinación es el elemento más antiguo en la organización estatal. Pensemos por ejemplo en aquel sistema de pesos y contrapesos que ya Montesquieu sostenía era imprescindible entre los poderes. Desde la perspectiva del concepto en estudio, puede sostener que estos frenos entre Poderes -si bien más visibles en sistemas parlamentarios puros-, no son sino mecanismos de coordinación necesaria entre ellos, buscando fortalecer la competencia o especialización de cada uno.

\section{III.5 INFORMES}

Existen diversos artículos que contienen vocablos que comiencen con «inform».

Así los artículos 118 y 119, como 284 y 285 refieren a informes como medio de control entre los Poderes Legislativo y Ejecutivo, tanto a nivel nacional como departamental. Quizás esto pueda valorarse más como coordinación y control que como informe técnico. Sí tenemos informes, en el sentido de opiniones técnicas, en los artículos 221 y 225 (artículo que por cierto, también menciona los empleos, para prohibir en el caso su creación).

La competencia atribuida al Tribunal de Cuentas y su potestad de contralor en general se expresa mediante informes. Así por ejemplo, los artículos 301 y 302 de la Constitución y sin dudas el 211. En agregado, la Junta Departamental podrá requerir su informe, conforme lo dispuesto por el numeral cuarto del artículo 273.

En relación con el Poder Ejecutivo, el numeral quinto del artículo 168 establece que le corresponde «informar al Poder Legislativo, al inaugurarse las sesiones ordinarias, sobre el estado de la República y las mejoras y reformas que considere dignas de su atención». Si bien el término es informar, quizás pueda verse esto como un caso de peso y contrapeso mencionado. 
Este concepto del acrónimo es quizás el más dudoso de ser aplicado a la constitución. Si bien el mismo es en realidad más amplio de lo que puede parecer en una primera lectura, nótese que los casos encontrados en el texto constitucional refieren a pedidos de informes que traducen una forma de ejercer control de unas organizaciones a otras.

\section{III.6 PRESUPUESTOS}

En este caso la búsqueda fue realizada por «presupuest».

El artículo 77 numeral 7 encabeza la lista de aquellos artículos que utiliza un vocablo que comienza de esa manera, declarando que no se requiere mayoría especial para la aprobación de la ley que resuelva en materia «de gastos, presupuestos y de orden interno de» la Corte Electoral y corporaciones electorales.

El ya mencionado artículo 65, al establecer las comisiones representativas de los personales respectivos, indica que estas tendrán entre sus fines el estudio del ordenamiento presupuestal, por lo que este artículo también se incluye en este aspecto del POSDCORB.

Es competencia de la Asamblea General, conforme el artículo 85 numeral 13 el «aprobar, reprobar o disminuir los presupuestos que presente el Poder Ejecutivo», existiendo además determinadas potestades que deben ser ejercidas mediante las leyes de presupuesto, conforme lo establecido por el artículo 86 ya mencionado. Esto se relaciona con la competencia otorgada al Poder Ejecutivo en el numeral 19 del artículo 168.

Por otra parte, conforme el artículo 108 «cada Cámara aprobará, dentro de los doce primeros meses de cada Legislatura, sus presupuestos por tres quintos de votos del total de sus componentes y lo comunicará al Poder Ejecutivo para que los incluya en el Presupuesto Nacional. Estos presupuestos se estructurarán por programas y se les dará, además, amplia difusión pública».

Previsiones similares resultan el artículo 239 para el Poder Judicial, los artículos 273 y 275 numeral 3 para la Junta Departamental y el Intendente, el artículo 321 con relación al Tribunal de lo Contencioso Administrativo y el 323 con la Corte Electoral.

Vemos en este caso, que para el presupuesto se establecen especialmente los procedimientos necesarios para su aprobación y modificación.

Particular atención merecen en este ítem de presupuestos los artículos 214 a 232. 
El contenido del Presupuesto Nacional no queda librado al azar, sino que todo lo contrario, el mismo está específicamente regulado en el artículo 214. Las competencias del Poder Legislativo están claramente delimitadas regulándose con detalle el proceso especial que conlleva la aprobación de la ley que lo contiene -el que se aparta del proceso de elaboración de la ley al que podemos llamar ordinario.

A nivel Departamental, nos encontramos con previsiones muy similares, estando también limitada las competencias de las Juntas Departamentales, en el artículo 225 ya mencionado.

En los artículos 220 y 221 nuevamente podemos ver cómo la actividad condiciona la organización y de ésta se condiciona su forma de aprobar presupuestos.

Del artículo 230 parece surgir que -no solo la planificación es esencial, al punto de condicionar la estructura- sino también el presupuesto, dado que como comentábamos más arriba, la Constitución prevé la existencia de un organismo, que ya su nombre indica, tendrá esta especialidad.

La disposición transitoria y especial letra E -ya mencionada- refiere a los presupuestos de las Direcciones que se mencionaba se creaban, facultando al Poder Ejecutivo para «tomar de Rentas Generales las cantidades necesarias para la instalación y funcionamiento de los referidos Ministerios, hasta que la ley sancione sus presupuestos de sueldos, gastos e inversiones». También utiliza el vocablo presupuesto justamente para referirse a la Comisión de Planeamiento y Presupuesto, la disposición transitoria y especial letra O.

La tan particular disposición letra $\mathrm{V}^{\prime}$ declara la inconstitucionalidad de «toda modificación de seguridad social, seguros sociales, o previsión social (Art. 67) que se contenga en leyes presupuestales o de rendición de cuentas, a partir del $1^{\circ}$ de octubre de $1992 »$, estableciendo los mecanismos para lograr el accertamento de la Suprema Corte de Justicia para obtener la eliminación de sus efectos. Puede observarse en este caso que la materia presupuestal condiciona el proceso de inconstitucionalidad al punto en que el constituyente estableció uno diferenciado para normas relacionadas a esta temática.

\section{III.7 ORGANIZACIÓN}

Hemos dejado organización para el final dado que al realizar la búsqueda para «organiz», los resultados son más diversos.

En primer lugar, se encuentra el artículo 50, que -en referencia a las actividades industria- 
les y comerciales y al fomento de las mismas-, establece que «toda organización comercial o industrial trustificada estará bajo el contralor del Estado». Como puede apreciarse, no se refiere aquí a organización en el sentido estudiado sino como sinónimo de empresa.

Encontramos luego el artículo 57 que establece: «La ley promoverá la organización de sindicatos gremiales, acordándoles franquicias y dictando normas para reconocerles personería jurídica». En este caso organización parecería ser sinónimo de creación.

Similar situación se presenta con el artículo 80, el que al establecer las causales de suspensión de la soberanía incluye en su numeral sexto el «formar parte de organizaciones sociales o políticas que, por medio de la violencia, o de propaganda que incitase a la violencia, tiendan a destruir las bases fundamentales de la nacionalidad». Organizaciones aquí parece referir más a grupos o sección social, pero no a la organización estatal.

El artículo 65 sí parece emplear el término en el sentido estudiado al establecer que «la ley podrá autorizar que en los Entes Autónomos se constituyan comisiones representativas de los personales respectivos, con fines de colaboración con los Directores para el cumplimiento de las reglas del Estatuto, el estudio del ordenamiento presupuestal, la organización de los servicios, reglamentación del trabajo y aplicación de las medidas disciplinarias». E incluso sin utilizar el mismo término, pero condicionando la estructura continúa «En los servicios públicos administrados directamente o por concesionarios, la ley podrá disponer la formación de órganos competentes para entender en las desinteligencias entre las autoridades de los servicios y sus empleados y obreros; así como los medios y procedimientos que pueda emplear la autoridad pública para mantener la continuidad de los servicios».

El artículo 67 establece por su parte que «las jubilaciones generales y seguros sociales se organizarán en forma de garantizar a todos los trabajadores, patronos, empleados y obreros, retiros adecuados y subsidios para los casos de accidentes, enfermedad, invalidez, desocupación forzosa, etc.; y a sus familias, en caso de muerte, la pensión correspondiente». Este artículo tiene clara relación con el artículo 195 ya mencionado, dado que el otro cometido del BPS además de coordinar es «organizar la seguridad social».

La estructura está claramente determinada para los casos de ciertos servicios. Conforme el artículo 186 «los servicios que a continuación se expresan: Correos y Telégrafos, Administraciones de Aduanas y Puertos y la Salud Pública no podrán ser descentralizados en forma de entes autónomos, aunque la ley podrá concederles el grado de autonomía que sea compatible con el contralor del Poder Ejecutivo». 
Encontramos además creado constitucionalmente el Banco Central de la República el que -conforme el artículo 196-, «estará organizado como ente Autónomo y tendrá los cometidos y atribuciones que determine la ley aprobada con el voto de la mayoría absoluta del total de componentes de cada Cámara».

Por otra parte, el artículo 213 ya mencionado, en relación con la presentación al Poder Ejecutivo del proyecto de Ley de Contabilidad y Administración Financiera elaborado por el Tribunal de Cuentas, establece que este «comprenderá las normas reguladoras de la administración financiera y económica y especialmente la organización de los servicios de contabilidad y recaudación; requisitos con fines de contralor, para la adquisición y enajenación de bienes y contratación que afecten a la Hacienda Pública; para hacer efectiva la intervención preventiva en los ingresos, gastos y pagos; y las responsabilidades y garantías a que quedarán sujetos los funcionarios que intervienen en la gestión del patrimonio del Estado».

El artículo 262 -ya mencionado- interesa aquí al análisis desde dos aspectos.

Por una parte, su penúltimo inciso utiliza el término organización al referirse a que «los Gobiernos Departamentales podrán acordar, entre sí y con el Poder Ejecutivo, así como con los Entes Autónomos y los Servicios Descentralizados, la organización y la prestación de servicios y actividades propias o comunes, tanto en sus respectivos territorios como en forma regional o interdepartamental».

Por otra, regula en concreto la organización dada a los Gobiernos Departamentales, estableciendo como estructura necesaria la existencia de una Junta Departamental y un Intendente, así como la posible existencia de autoridades locales.

\section{COMENTARIOS FINALES}

Baste este somero análisis para demostrar que la Constitución puede ser leída a la luz de las categorías del POSDCORB.

Si bien es este un primer estudio - quedan aún varios artículos por categorizar-, en muchos casos ya puede verse cómo estas acciones condicionan la estructura estatal y en otros como ésta condiciona aquellas.

Se ve reflejado además que la llamada parte dogmática de la Constitución -si bien en menor medida que la orgánica-, también presenta artículos que pueden leerse bajo el concepto analizado. 
Lo descripto sin embargo, no podría causarnos mayor asombro. Después de todo, ya el primer artículo de la Carta implica una organización entre todos, una organización a la que llamamos República Oriental del Uruguay.

\section{BIBLIOGRAFÍA CONSULTADA}

DELPIAZZO, C. (SF). Bases de la organización administrativa. Retos de la organización administrativa contemporánea, páginas 821-843.

FAYOL, H. (1961). Administración industrial y general. México DF: Editorial Intercontinental S.A.

GUERRERO, O. (1997). Principios de la administración pública. Santa Fe de Bogotá: Unidad de Publicaciones ESAP. Disponible en: http://www.iapqroo.org.mx/website/ biblioteca/PRINCIPIOS\%20DE\%20LA\%20ADMINISTRACION\%20PUBLICA.pdf (consultado por última vez 25/10/2016).

RAMIÓ, C. (SF). Teoría de la organización y administración pública. Disponible en: http://www.fcpolit.unr.edu.ar/tecnologiasdelaadministracion/files/2012/08/U1-Carles-Ramio-TeoriA-de-la-Organizacion.pdf (consultado por última vez 25/10/2016).

RIASCOS, J. (SF). Teoría clásica de la administración. Disponible en: https://www. google.com.uy/url? sa $=$ t\&rct $=\mathrm{j} \& \mathrm{q}=\&$ esrc $=$ s\&source $=$ web \& $\mathrm{cd}=7 \& \mathrm{cad}=\mathrm{rja} \& u a c t=8 \&-$ ved=0ahUKEwiau6a8ivfPAhUC9WMKHcJ2DB8QFgg9MAY\&url=http $\% 3 \mathrm{~A} \% 2 \mathrm{~F} \%$ 2Fcmap.upb.edu.co\%2Frid\%3D1157507875371_986871460_1592\%2Fteoclasi.ppt\&usg=AFQjCNErTUT8c4U98SPyodDOaqthwcyV-w\&bvm=bv.136593572,d.cWw (consultado por última vez 25/10/2016).

RODRÍGUEZ, J. (1999). Las teorías clásicas de la organización. Disponible en: http:// www.insumisos.com/lecturasinsumisas/LAS\%20TEORIAS\%20CLASICAS\%20 DE\%201a\%20ORGANIZACION.pdf (consultado por última vez 25/10/2016).

ROTONDO, F. (2015). Incidencias de las nuevas tecnologías en la organización administrativa: la gobernanza electrónica. En Rotondo F. (1era edición), Organización administrativa, páginas 127-149.

TAYLOR, F. (1975). Principios de la administración científica. Buenos Aires: Editorial 


\section{El Ateneo.}

URWICK, L. (1967). Los elementos de la administración. 4ta edición en español. Palazón, R. (traductor). Mexico: Herrero Hermanos Sucs S.A.

Fecha de recepción: 18 de abril 2020.

Fecha de aceptación: 30 abril 2020. 